\title{
Eclampsia in a Dog : An Overview
}

\author{
M. M. Pathan*, G. M. Siddiquee, A. Latif, H. Das, Md. J. Z. Khan and M. K. Shukla, \\ Dept. of Animal Physiology \& Biochemistry \\ College of Veterinary Science \& A. H. Sardarkrushinagar Dantiwada Agricultural University, \\ Sardarkrushinagar - 385 506, India. \\ * Corresponding author: Tel. 91-9541337806, E-mail: drmohsinvets@gmail.com
}

\begin{abstract}
Eclampsia is an acute, life-threatening disease caused by low blood calcium levels (hypocalcaemia) in dogs and more rarely in cats. The causes of Eclampsia are poor nutrition, low blood level of albumin, excessive milk production and disease of parathyroid gland. Imbalance between the rates of in flow and out flow from the extra cellular fluid calcium because of the increased loss into the milk appears to be an important factor in the pathogenesis of puerperal tetany in the bitches. Normally the condition is diagnosed by careful investigation, recording proper history, correlating the clinical signs, response to therapy in most cases and confirming the condition with laboratory diagnosis. Generally, 5-10 ml of $10 \%$ calcium gluconate will provide sufficient calcium for a bitch weighing between five and ten $\mathrm{Kg}$. Dietary supplements of calcium and vitamin $\mathrm{D}$ are useful in preventing relapse of the disease after treatment and prevention of the disease.
\end{abstract}

Keywords: Hypocalcaemia, Milk production, Dietary Supplementation, Malnutrition.

\section{I ntroduction}

Eclampsia is an acute, life-threatening disease caused by low blood calcium levels (hypocalcaemia) in dogs and more rarely in cats. There is lesser documentation of hypocalcaemia in dogs and cats as compared to cattle (Rowland et al., 1972). Canine Eclampsia more correctly called Puerperal Tetany is a startling and dangerous condition brought on by extremely low levels of calcium in the blood stream. It is sometimes erroneously called as milk fever. Low blood calcium is the result of reduction of calcium levels outside the cells of the bitch's body. The disease most commonly occurs in bitches 1-3 weeks after giving birth but the cases are also sometimes observed during gestation. As the demand for milk increases, the likelihood of eclampsia escalates. Normally, by 40 days after whelping, the danger of eclampsia has passed. The pups of the affected bitches may not be usually affected as the affected bitches usually produce milk with normal calcium level. Lctating animals are especially susceptible to blood calcium depletion because of milk production. The body of some lactating dogs and cats simply cannot keep up with the increased demands for calcium. Animals with Puerperal Tetany lack the ability to quickly move calcium into their milk without depleting their own blood levels of this mineral. Smaller breeds of dogs are more prone to the disease as compared to the larger breeds. The disease has been reported to be more frequently observed in small, hyper excitable breeds of dogs. Eclampsia may not be necessarily associated with large litter size.

\section{Etiology}

This disease condition can be produced by hypocalcaemia (low blood calcium), which can be brought on by the following:

1. Poor Nutrition - In most of the cases the dog owners are not aware of the nutritional requirements of their dog and may either feed diet deficient in nutrients or feed nutrients in excess. "Home brewed" diets are usually common predisposing factor for this disease. The owner innocently may be adding too much unbalanced meat to the bitch's diet, thinking that the extra protein is beneficial. This normally disturbs the calcium to phosphorus ratio because the amount of useful calcium in the food is actually reduced. The ideal diet for a dog should have calcium: phosphorus ratio of 1.2 to 1 . Many organ meats such as liver have calcium to phosphorus ratio of 1 to 15 . Liver is great for dogs but if it comprises a large part of the diet, the calcium/phosphorus ratio of the diet will be improper.

2. Low Blood Levels of Albumen - Dietary protein deficiency or excessive loss of albumen from the body 
will cause low levels of calcium. This happens in some kidney diseases.

3. Excessive Milk Production - When pups require large amounts of milk (10 to 30 days post whelping) the bitch's ability to maintain proper amounts of calcium in her blood stream becomes stressed. As pups grow their total daily intake of milk increases which puts a greater and greater demand on bitch's milk production. Interestingly, even with small litters, some bitches produce so much milk so rapidly that their blood calcium levels cannot be maintained. Milk production has priority over the blood stream for calcium. Since calcium ions in the blood have a dynamic impact on nerve transmission and muscle contractility, all sorts of physiologic dysfunction will arise if blood calcium levels are too low.

4. Disease of the Parathyroid Glands - The diseases of the parathyroid glands are also believed to cause hypocalcaemia in dogs and cats. This condition is quite rare.

\section{Patho-physiology}

Imbalance between the rates of in flow and out flow from the extra cellular fluid calcium because of the increased loss into the milk appears to be an important factor in the pathogenesis of puerperal tetany in the bitches. Severe hypocalcaemia and often hypophosphatemia develop near the time of peak lactation (approximately one to three weeks postpartum); probably as the result of an imbalance between the rates of inflow and outflow from the extracellular calcium pool (Ettinger, 1983). There is excessive loss of stabilizing membrane bound calcium resulting in increased permeability of nerve membranes to ions. These membranes, therefore, require stimulus of lesser magnitude to depolarize. Functional disturbances associated with hypocalcaemia in the bitch are due to neuromuscular tetany. Tetany occurs in bitches as a result of spontaneous repetitive firing of motor nerve fibers.

The addition of calcium supplements or diets excessively high in calcium may exert a negative biofeedback on the secretion of hormones by the parathyroid glands that control calcium metabolism. This effect can cause a decrease in both the body's capabilities to mobilize calcium stores from bone and its ability to increase calcium absorption in the intestine. When calcium is suddenly needed for lactation, the body's regulatory mechanisms are unable to adapt quickly enough to the sudden calcium loss. Since calcium is diverted to milk production, the bitch's blood calcium levels decrease. Feeding high calcium diet to dairy cows in the prepartum period also has provocative effect on development of hypocalcaemia due to decreased responsiveness of PTH-mediated bone resorption. The resulting decreased availability of calcium from skeletal sources leads to increased reliance on intestinal calcium absorption.

\section{Symptoms}

Eclampsia is a very serious disorder but fortunately the signs are fairly easy to recognize, especially when it is coupled with late term pregnancy and/or milk production. Initially, the affected dog will be restless and nervous. Within a short time, she will walk with a stiff gait and may even wobble or appear disoriented. Eventually, the bitch may be unable to walk and her legs may become stiff or rigid. Muscle tremors, eye twitching, panting, in coordination may also be observed. The bitch may have fever; with body temperature even over $105^{\circ} \mathrm{F}$. Temperature up to $107^{\circ} \mathrm{F}$ is also not uncommon (Ettinger, 1983). This rise of temperature is often associated with increased muscular activity. The respiration rate will also increase. At this point, death can occur if no treatment is given. The clinical course of the disease is very rapid and the bitch may proceed from the initial symptoms of restlessness, panting and nervousness to ataxia, trembling, muscle tetany and convulsive seizures within 12 hours (Resnik, 1972).

\section{Diagnosis}

Normally the condition is diagnosed by careful investigation, recording proper history, correlating the clinical signs, response to therapy in most cases and confirming the condition with laboratory diagnosis. The bitches suffering of eclampsia have a history of whelping one to two weeks ago, sudden start of pacing and hesitation to nurse. The animal may show the signs of panting, shaking all over, and it may not be able to even stand up. Laboratory examination may be performed for the diagnosis of Eclampsia by estimation of Serum calcium, Serum phosphorus and Blood glucose levels. In canines affected with eclampsia, the serum calcium level is less than 7 $\mathrm{mg} / \mathrm{dl}$. The Serum phosphorus levels are often lowered. The Blood glucose levels are in the low normal range or decreased due to severely increased muscular activity as a result of tetany. Eclampsia is generally associated with proteinuria (Vigue, 1953).

\section{Treatment}

Eclampsia can be treated by a slow and careful intravenous injection of a calcium solution under the $d$ 
close supervision of the veterinarian. Generally, 5-10 $\mathrm{ml}$ of $10 \%$ calcium gluconate will provide sufficient calcium for a bitch weighing between five and ten $\mathrm{Kg}$. Calcium should be administered slowly to avoid ventricular fibrillation and cardiac arrest. Often a patient needs to be monitored through ECG or other device to record heart rate and blood pressure. After only a few minutes of seizure and even continuous tremors or spasms, the patient's temperature will rise to dangerously high values. It isn't uncommon for a $\mathrm{dog}$, whose normal body temperature is generally near 101 and $102^{\circ} \mathrm{F}$, to have a body temperature of $107^{\circ} \mathrm{F}$. Any higher values will surely lead to irreversible brain damage. Cool bath should be given, in such cases, to lower body temperature to normal. After only a few minutes of muscle spasms the bitch will be thoroughly exhausted and rapidly available glycogen (glucose) stored in the liver and muscles can be depleted. Low blood sugar can trigger seizure activity, too. Therefore, intravenous injection of dextrose for quick energy should be given to eclampsia patients. If the tremors are severe mild sedation or use of phenobarbitone may be beneficial to assist in relaxing the muscles.

Dietary supplements of calcium and vitamin D are useful in preventing relapse of the disease after treatment. Some clinicians prefer using corticosteroids in addition to calcium and vitamin D. Administration of steroids like Prednisolone $(2.5 \mathrm{mg}$ tablet BID for 3 days followed by $1.25 \mathrm{mg}$ tablet BID for 3 days and then $1.0 \mathrm{mg}$ tablet BID for 7 days) has also been advocated for the treatment of Eclampsia (Chakraborty, 2006). But contradictory literature questioning the efficacy of corticosteroids in eclampsia (Ettinger, 1983) is also on documents as corticosteroids may lower serum calcium level by interfering with intestinal calcium transport.

\section{Prevention}

Once a dog has had milk fever, there is an excellent chance that she will also have it with future litters if preventive steps are not taken. Therefore, suitable preventive measures need to be taken to prevent this disease. The following preventive measures can be taken for prevention of Eclampsia:

1. Provide balanced diet with proper Calcium Phosphorus ratio during gestation. High quality meat-based balanced food may be provided. Don't over supplement with all sorts of calcium, vitamins or meat products.

2. Give only small amount of a balanced source of calcium, phosphorus, and Vitamin D to the bitch's diet beginning about mid-term. Milk makes a good "supplement" as long as the lactose does not create loose stool. Do not add calcium alone, it must be used with phosphorus and Vitamin D. Remember that optimum ratio of calcium to phosphorus is 1.2 to 1 .

3. Cortisone supplement prior to whelping are helpful in preventing Canine Eclampsia.

4. Supplement the puppies' with a milk replacer as soon as possible to decrease the milk demands on the bitch. Preferably, the pups of the susceptible bitches should be removed from the bitch for 24 hours and fed on milk substitute or other suitable diet. Commercial milk replacers are also available.

5. Wean the pups as soon as possible.

\section{References}

1. Chakraborty, A. (2006). Dogs Their Care and Treatment. 3rd revised edn., Kalyani publisher, Luthiana.

2. Ettinger, S. D. (1983). Textbook of Veterinary internal medicine-Diseases of dogs and cats, 2nd edn., WB Saunders Company

3. Resnik, S. (1972). Hypocalcaemic tetany in the dog. $J$. Am. Vet. Med.Assoc. 144: 1155

4. Rowland, G. N., et.al.(1972). Microradiographic evaluation of bone from cows with experimental hypovitaminosis $\mathrm{D}$, diet induced hypocalcaemia and naturally occurring parturient peresis. Tissue Res. 9: 179.

5. Vigue, R. F. (1953). Vet Med. 48, 70. (C. f.) Lactation Vol II Biosynthesis and secretion of milk /diseases. Larson, B. L. and Smith, B. R. (1974). Academic Press, New York. 